\title{
BMJ Open Exploring network structure and the role of key stakeholders to understand the obesity prevention system in an Australian metropolitan health service: study protocol
}

\author{
Jonine Jancey, ${ }^{\oplus 1,2}$ Justine Elizabeth Leavy, ${ }^{\circledR 1,2}$ Christina Pollard, ${ }^{3}$ Therese Riley, ${ }^{4}$ \\ Maria Szybiak,, ${ }^{5,6}$ Megan Milligan, ${ }^{3}$ Dan Chamberlain, ${ }^{4,7}$ Krysten Blackford ${ }^{\odot, 2}$
}

To cite: Jancey J, Leavy JE, Pollard C, et al. Exploring network structure and the role of key stakeholders to understand the obesity prevention system in an Australian metropolitan health service: study protocol. BMJ Open 2019;9:e027948. doi:10.1136/ bmjopen-2018-027948

- Prepublication history for this paper is available online. To view these files, please visit the journal online (http://dx.doi. org/10.1136/bmjopen-2018027948).

Received 15 November 2018 Revised 21 March 2019 Accepted 30 April 2019

Check for updates

(C) Author(s) (or their employer(s)) 2019. Re-use permitted under CC BY-NC. No commercial re-use. See rights and permissions. Published by BMJ.

For numbered affiliations see end of article.

Correspondence to Dr Krysten Blackford; k.blackford@curtin.edu.au

\section{ABSTRACT}

Introduction Little progress has been made to address the increasing obesity prevalence over the past few decades, and there is growing concern about the farreaching consequences for health and well-being related to obesity on a global scale. Systems thinking is emerging as a suitable approach for obesity prevention, as it allows health researchers, practitioners and policy-makers to systematically synthesise existing data, expose gaps, inform priority setting and identify leverage points in the system. The aim of this study is to trial a systems thinking approach to better understand the local obesity prevention system, and identify gaps and viable opportunities for health promotion activities to strengthen obesity prevention efforts in an Australian metropolitan health service.

Methods and analysis A mixed methods design will be undertaken in a metropolitan health service area in Perth, Western Australia in 2019-2020. A systems inventory audit will be used to identify physical activity, nutrition and overweight/obesity prevention activities taking place in the study area. An organisational network survey will be administered, and a social network analysis undertaken to examine relationships between organisations in the network. The relationships and interactions will compare the level and type of interactions each organisation has within the network. Parameters including density, centrality and betweenness will be computed using UCINET and Netdraw.

Ethics and dissemination Ethics approval has been obtained from the Curtin University Human Research Ethics Committee (approval number HRE2017-0862). Results will be reviewed with members of the advisory group, submitted to relevant journals and presented at relevant conferences to health promotion practitioners and policy-makers. The area health service, as co-producers of the research, will use findings to inform policy and strategy across the study area.

\section{INTRODUCTION}

\section{Health issue}

The prevalence of obesity is increasing across the globe, and with almost two in three Australian adults and one in four Australian

\section{Strengths and limitations of this study}

- The research will identify obesity prevention programmes, collaborations and interactions, to help identify gaps in the system and directly inform decision making processes to strengthen obesity prevention efforts.

- The quality of data will depend on the willingness of participants and organisations in the study area to share information.

- De-identification of organisation data may make planning and improvements in the health service area challenging.

- The co-production approach addresses the usual gap between research, policy makers and practitioners' needs, creating timely, real-world outcomes.

- The suitability of this process will be evaluated for duplication and translation into other priority areas for health services and local government (eg, tobacco control; alcohol harm reduction).

children overweight or obese, Australia has some of the highest recorded rates of obesity in the world. ${ }^{1}$ In 2014-2015, approximately $63 \%$ of Australian adults were overweight or obese which is equivalent to approximately 11 million people. ${ }^{2}$ Little progress has been made addressing the issue over the past few decades, and there is growing concern about the far-reaching consequences for the health and well-being of populations. ${ }^{3}$

As the prevalence of overweight and obesity increases, health and welfare costs will also increase substantially. ${ }^{4}$ The financial consequences for the individual and the healthcare system will adversely impact workplace productivity, participation in the workplace and economic growth. ${ }^{5}$ In 2010, the direct costs associated with obesity and associated chronic disease in Australia was calculated to be $\$ 21$ billion annually and indirect 
costs $\$ 35.6$ billion, deriving an overall annual cost of $\$ 56.6$ billion. ${ }^{6}$ This ever increasing annual cost further escalates obesity as a public health priority and highlights the need for effective and comprehensive prevention efforts. $^{7}$

Obesity is a 'complex problem' and innately challenging. Unlike simple problems where outcomes of actions are linear and can be predicted, complex problems involve multiple actors and require multiple interventions that go beyond the focus of personal responsibility. ${ }^{8}$ Halting and reversing obesity both in Australia and globally will require changing our societal approach to consumption of food, beverages, and physical activity behaviours ${ }^{3}$ which are part of complex systems that include food supply, communications, transport, urban design, business, sociocultural, education, health, trade, economic, governance, urban design and marketing systems. ${ }^{9}$ Understanding these complexities has supported the emergence of systems thinking as an obesity prevention approach. $^{1011}$

\section{Systems thinking}

To address the problem of obesity, it is imperative to understand the community system in which it occurs, and implement health promotion interventions that involve coordinated interconnected components that consider physical, biological, ecological, social, political and organisational relationships. ${ }^{12}$ Accordingly, systems thinking recognises the characteristics of these relationships and emphasises the non-linear, dynamic and adaptive nature of the overall system. ${ }^{13}$ Systems thinking tools and methods allow researchers and decision-makers to examine a system's components and levels, which can assist with planning appropriate interventions. ${ }^{14} 15$

Systems thinking has been used in other disciplines such as physics, economics, engineering and systems biology, ${ }^{16}$ and has more recently been introduced to the public health field to address a wide range of health problems. ${ }^{14}$ A major reason for the recent adoption of systems thinking in health promotion is the growing recognition of its ability to inform investments and actions that address complex problems in specific domains (eg, neighbourhood effects on health and obesity). Often there are a number of similar programmes and activities operating simultaneously within a community, coordinated and funded by an array of organisations and funding bodies. Therefore, the systems thinking approach provides health researchers, practitioners and policy-makers with the tools to systematically synthesise existing data, expose gaps, inform priority setting and identify leverage points in the system. ${ }^{16-18}$

Social network analysis (SNA) is a tool that is commonly used in systems thinking to transform health practice. ${ }^{19}$ SNA explores the structures and processes of a network which may constitute individuals or organisations (actors) that are linked by ties to one another. ${ }^{20}$ This type of analysis focuses on relationships within a network rather than studying the attributes of individuals or organisations that comprise the network. ${ }^{20}$ It can identify what ties actors have and whether they are informal (eg, an organisation is known to another organisation), or formal (eg, funding or joint-partnership of service delivery) ${ }^{21}$ Understanding the types and functions of networks ${ }^{21}$ and what role key actors play is essential to understand if, where, and how to intervene in a system.

\section{Western Australian context}

The Western Australian (WA) Health Promotion Strategic Framework ${ }^{22}$ is a state government framework that adopts a whole-of-population approach to addressing obesity, with identified targeted interventions aimed at reducing health inequalities in communities who have a high prevalence of the associated chronic disease risk factors. The WA Department of Health has developed a 5-year plan to curb the rise of overweight and obesity in the WA population and has highlighted the need for interventions to take a comprehensive approach to prevention. ${ }^{22}$ In support of this comprehensive approach, a large and newly formed government Health Service in metropolitan Perth WA has been established which is an extensive hospital and health service network that aims to maintain and improve the health and well-being of more than 708000 residents in its catchment area across 13 local government areas (LGAs).

There is dearth of information regarding the organisations and networks that operate within the area health service that address obesity, physical activity and nutrition to improve long term health outcomes. Using a collaborative approach and systems thinking informed methods previously trialled in a range of communities across Australia, ${ }^{23}$ a partnership between a university, non-government organisation and government department was established to undertake this project. This collaborative research aims to better understand the obesity prevention system located in a metropolitan area of WA. This exploratory research will use a systems thinking approach to better understand the local obesity prevention system defined by the geographical catchment of an area health service; and identify potential gaps and viable opportunities for health promotion investments that will improve obesity prevention efforts and actions.

\section{METHODS AND ANALYSIS}

This mixed methods study, which uses systems thinking tools, will be conducted in 2019-2020 and comprises three phases. It will explore the boundaries, networks and actions of the local obesity prevention system in the study area, which includes the 13 LGAs that comprise the area health service. The study design is based on previous studies $^{24-26}$ and methods undertaken in Australia ${ }^{23}$ 27-29; and makes use of The Systems Change Framework ${ }^{30}$ and its iterative inquiry process involving four stages: (1) define the situation, (2) gain clarity, (3) find leverage and (4) act strategically. These key elements will enable a deeper understanding of the obesity prevention system 
in the study area. This type of inquiry involves an ongoing process of interrogation to shift from the current undesirable state to a future desirable state.

\section{Primary aim}

This exploratory research will use a systems thinking approach to better understand the local obesity prevention system defined by the geographical catchment of an area health service; and identify potential gaps and viable opportunities for health promotion investments that will improve obesity prevention efforts and actions.

\section{Research objectives}

- Profile the chronic disease and health risks relevant to overweight/obesity for the study population (phase $1)$.

- Explore local physical activity, nutrition and overweight/obesity prevention activities taking place in the study area (phase 2).

- Identify the most influential stakeholders, collaborations and activities within the study area (phase 3).

- Identify opportunities to support these actions or make system improvements (phase 4).

\section{Advisory group}

An advisory group comprising the research team and selected experts in obesity prevention will be formed in the early stages of the project. The research team will identify and invite experts to join the advisory group who have relevant experience in health promotion policy and practice, including relevant stakeholders and practitioners with current expertise in non-government organisations, local government and Aboriginal and Torres Strait Islander issues. The group will meet on a quarterly basis for 2 years to identify relevant stakeholders and potential collaborators; review research instruments as required; consider findings and provide feedback; and make recommendations for research translation.

\section{Phase 1: LGA profiling \\ Aim}

To describe the context of the study area by identifying the preventable risk factors for overweight/obesity, and prevalence of associated chronic diseases (such as type 2 diabetes mellitus, cardiovascular disease and some cancers) for each LGA in the study area. All population groups will be included in the summary.

\section{Method}

This phase is based on the Prevention Tracker community snapshot method ${ }^{27}$ and relates to stage one of the iterative inquiry process of The Systems Change Framework ${ }^{30}$ whereby enabling factors and initial boundaries for the systemic inquiry are identified. Micro (behavioural) and meso (institutional) obesity risk factors will be explored, ${ }^{31}$ specifically poor nutrition, inadequate physical activity and excess body weight. The research team and advisory group will outline the scope and nature of the health profiles of the 13 LGAs. The Health Services health promotion team will develop a set of health indicators which will be collated from departmental monitoring systems and other sources. All data will be provided to the advisory group and research team for use in phase 2 .

\section{Phase 2: System inventory}

Aim

To identify current physical activity, nutrition and overweight/obesity prevention activities taking place in the study area. These data will be used to refine the approach to phase 3 .

\section{Method}

This phase also relates to stage one of the iterative inquiry process of The Systems Change Framework ${ }^{30}$ and will be used to identify factors to facilitate systems change efforts. The System Inventory method ${ }^{28}$ will be adapted by the research team for the study area. The inventory will capture a comprehensive list of the key organisations that contribute to prevention efforts across the study area.

\section{Participants and sampling}

Participants will include health promotion practitioners, policy makers and other health professionals involved in the delivery of physical activity, nutrition and obesity prevention programmes and activities in the study area. The advisory group and health promotion officers employed by the area health service will use Nominal Group Technique $^{32}$ to reach consensus on the key organisations and individuals to be interviewed initially, based on the inclusion/exclusion criteria outlined below. All potential organisations will be sent a letter inviting them to participate in a face-to-face interview with two health promotion officers. Interested participants will be provided with a study information sheet, and informed written consent will be obtained prior to the interview commencing. To ensure that all relevant individuals are included, a referral sampling technique will be used whereby participants can nominate other relevant stakeholders and individuals involved in physical activity, nutrition or obesity prevention in the study area. ${ }^{33}$

\section{Data collection}

A custom-built database will be designed using Microsoft Access software to record details of prevention activities being employed in the study area. The content of the database will be based on the Systems Inventory instrument, ${ }^{28}$ and the database will be reviewed by the research team to confirm face and content validity. It will then be trialled with health professionals $(n=10)$ and changes made as required. The health promotion officers $(n=6)$ will then review and trial the final instrument for usability, suitability and comprehension. Training workshops for the health promotion officers on data collection and use of the database will be conducted in conjunction with the instrument trial.

The database will capture information for each activity/programme delivered by participants and the organisations they represent. The inventory will include 
information on the: organisation type; contact information, overarching programme objectives and strategies, types of activities implemented (physical activity, nutrition and obesity prevention), activity duration, collaborating partners, target population, settings/locations the programme/activity operates, programme evaluation and programme funding details. Activities will be included in the system inventory if they are: (a) a current intervention strategy (policy/regulation or programme), (b) conducted in or reach the study area, (c) comprise more than one activity session to indicate an ongoing nature, (d) population-based or community-based and (e) aimed at obesity prevention, specifically nutrition, physical activity and/or overweight/obesity prevention. Activities will be excluded if they are: (a) delivered by alternative therapists based on Australian Health Practitioner Regulation Agency ${ }^{34}$ guidelines, (b) pharmacological interventions (special purpose dietary supplements), (c) one-on-one interventions/practitioners delivering individually focused programmes and (d) not comprising a core component (at least $75 \%$ ) focusing on nutrition, physical activity or overweight/obesity prevention. These data items will collectively make up the inventory of obesity prevention activities in the study area.

\section{Data analysis}

Descriptive statistics will be used to summarise the systems inventory data for the study area. All statistical analyses will be performed using SPSS Statistics Package V.25. ${ }^{35}$ Results will inform phase 3.

\section{Phase 3: Organisational network survey}

Aim

To identify the most influential stakeholders undertaking obesity prevention activities within the study area, examine the relationships between them, and identify potential system improvements.

\section{Method}

This phase relates to stages two and three of the iterative inquiry process of The Systems Change Framework ${ }^{30}$ and will support a deeper understanding of the system in terms of perspectives, relationships and boundaries; and find leverage by exploring opportunities for engaging in the system. An online organisational network survey will be undertaken to assess the degree of interrelatedness between a sub-sample of organisations identified during phase 2 . The survey has been previously developed and trialled $^{3637}$ and will be adapted for the current context. Relationships and interactions between the organisations will be captured via quantitative methods and analysed to provide a visual representation of the network structure in terms of interactions, activities and collaborations between organisations within the system.

\section{Participants and sampling}

Key decision-makers from a range of organisations will be sampled from the inventory produced during phase 2. Based on previous studies, ${ }^{29}$ the research team will identify the top 30 organisations nominated during the Systems Inventory. This will support achieving the required response rate of $75 \%$ for the network data to be considered reliable. ${ }^{38}$ Researchers will initially make telephone contact with the identified stakeholders to inform them of the research aim, and participants will be provided with a link to the survey. Participants will provide informed consent prior to commencing the survey. If there has been no response after 1 week, researchers will follow-up via telephone and offer assistance to complete the survey.

\section{Data collection}

Data will be collected via an online survey using Qualtrics. ${ }^{39}$ Participants will be asked to identify the organisations with which they share information, knowledge, or resources; engage in joint planning; apply for joint funding; and share informal contacts. Participants will be asked to choose from a compiled list of organisations, and will have the opportunity to expand on their responses using open-ended textboxes.

\section{Data analysis}

Data will be analysed using SNA which explores social phenomena through analysis of a network's structure. Data will be mapped and analysed using UCINET six for Windows ${ }^{40}$ and Netdraw software. ${ }^{41}$ Links between organisations will be mapped visually as a network graph, and the analysis will examine the role of organisations in the network relative to others by plotting the centrality scores. Core-periphery analysis will be conducted to identify densely connected core-nodes and sparsely connected periphery-nodes. ${ }^{42}$ Central organisations will be identified by degree centrality (most connected within the network), closeness centrality (connecting quickly to the whole network), and betweenness centrality (strategically placed in the network). ${ }^{43}$ Organisation characteristics will also be used to determine the basis of clustering. This will enable the identification of potential organisational collaborations, and gaps in the service delivery system across the study area. Nodes will be de-identified for presentation. The data will be available on contact when the researchers have agreed analysis is complete.

\section{Phase 4: System improvements}

At the completion of phase 3, The Systems Change Framework $^{30}$ will be revisited to complete the final stage of the iterative inquiry process. The purpose of this stage will be to respond to emerging system dynamics and engage in planning to address any gaps in the obesity prevention system.

\section{Patient and public involvement}

No patients were directly involved in setting the research question or the design of the study. Community representation has been obtained via the advisory group which provided guidance on study design. 


\section{ETHICS AND DISSEMINATION}

Participants will receive an information sheet stating that all data collected during each phase of the study will be deidentified and treated as confidential. Data will be password protected and saved to a secure server. A coordinated knowledge exchange via the advisory group and research agencies will be undertaken as part of the dissemination strategy. The research plan utilises push/pull knowledge exchange strategies to clearly describe how the findings are promoted and used. Push/pull knowledge translation in public health refers to the dissemination efforts by researchers (push) and the potential for limitation in its momentum by policy-maker and practitioner motivation and capability (pull). ${ }^{45}$ The push-pull framework will be used to guide community engagement and appropriate dissemination as outlined below.

\section{Push strategy}

It is intended that policy and action briefs for relevant stakeholders will be developed in partnership with the advisory group to provide an overview of the project, process findings and recommendations at the completion of each phase. Targeted presentations and skill building activities will be brokered; for example, stakeholder workshops to discuss findings, the delivery of agency-related conference presentations. Key findings will be prepared for dissemination and communicated appropriately to relevant stakeholders in government, non-government and community organisations. This may include using agency-specific communication methods through newsletters, social media, online and website advertising and content, as well as seminars as appropriate. Peer reviewed journal articles and fact sheets will be made available to build the capacity of the professional workforce in health, public health, government and non-government agencies, as well as the broader general community. Research reports will be made available through the research organisation websites, and potential replication and duplication of processes and uptake of findings will be supported.

\section{Pull strategy}

A key strength of the programme design is the utilisation of knowledge brokers (stakeholder managers and researchers) and programme champions (health promotion officers, local government staff and/or other relevant stakeholders) which will help build capacity in the professional workforce for the purpose, use and implementation of systems thinking tools and processes in the Perth metropolitan area, Western Australia. Although this current research focuses on the priority areas of nutrition, physical activity and overweight/obesity prevention, one of the aims of undertaking a prevention systems thinking approach is to determine the suitability of this process for other priority areas, such as, tobacco control or the drug and alcohol use. Determining the suitability of the current research methods for duplication and translation for other areas is a priority for health services and local government.

\section{Author affiliations}

${ }^{1}$ School of Public Health, Curtin University, Perth, Western Australia, Australia ${ }^{2}$ Collaboration for Evidence, Research and Impact in Public Health, Curtin University, Perth, Western Australia, Australia

${ }^{3}$ Population and Community Health, East Metropolitan Health Service, Perth, Australia

${ }^{4}$ The Australian Prevention Partnership Centre, Sax Institute, Haymarket, New South Wales, Australia

${ }^{5}$ Cancer Prevention and Research, Cancer Council Western Australia, Subiaco, Australia

${ }^{6}$ Public Health, Heart Foundation Western Australia, Subiaco, Australia ${ }^{7}$ School of Psychology and Public Health, La Trobe University, Melbourne, Victoria, Australia

Acknowledgements The authors would like to thank Cassandra Bordin for her continuous support during the process of this protocol development.

Contributors JJ, JL, CP, TR, MS, MM, DC and KB participated in the design of the study and data collection instruments. JJ and KB drafted the manuscript, and JJ, $\mathrm{JL}, \mathrm{CP}, \mathrm{TR}, \mathrm{MS}, \mathrm{MM}, \mathrm{DC}$ and $\mathrm{KB}$ read and approved the final manuscript.

Funding This project is part of the Healthway Health Promotion Exploratory Research Grants project 31980. East Metropolitan Health Service and Curtin University provided initial seed funding. The Prevention Tracker project was funded by the NHMRC, Australian Government Department of Health, NSW Ministry of Health, ACT Health and the HCF Research Foundation.

Competing interests None declared.

Patient consent for publication Obtained.

Ethics approval Ethics approval has been obtained from the Curtin University Human Research Ethics Committee (Approval Number HREC2017-0862).

Provenance and peer review Not commissioned; externally peer reviewed.

Open access This is an open access article distributed in accordance with the Creative Commons Attribution Non Commercial (CC BY-NC 4.0) license, which permits others to distribute, remix, adapt, build upon this work non-commercially, and license their derivative works on different terms, provided the original work is properly cited, appropriate credit is given, any changes made indicated, and the use is non-commercial. See: http://creativecommons.org/licenses/by-nc/4.0/.

\section{REFERENCES}

1. Australian Institute of Health and Welfare. A picture of overweight and obesity in Australia. Cat. no. PHE 216. Canberra: 2017 Contract No.: 978-1-76054-246-7.

2. Australian Institute of Health and Welfare. Healthy Communities: Overweight and obesity rates across Australia, 2014-15, 2016.

3. Kleinert S, Horton R. Rethinking and reframing obesity. Lancet 2015;385:2326-8.

4. National Health and Medical Research Council. Obesity and Overweight. 2015 www.nhmrc.gov.au/health-topics/obesity-andoverweight (cited 18 Jan 2018).

5. The Australian Prevention Partnership Centre.. Building a compelling case for prevention. 2017 http://preventioncentre.org.au/our-work/ research-projects/building-a-compelling-case-for-prevention/ (cited 201822 Jan)

6. Colagiuri S, Lee CM, Colagiuri R, et al. The cost of overweight and obesity in Australia. Med J Aust 2010;192:260.

7. National Health and Medical Research Council. Obesity and Overweight. 2017 https://www.nhmrc.gov.au/health-topics/obesityand-overweight (updated 2015).

8. Ulijaszek S. With the benefit of Foresight: Obesity, complexity and joined-up government. Biosocieties 2015;10:213-28.

9. Swinburn B, Dietz W. Kleinert S. A Lancet Commission on obesity. The Lancet 2015;386:1716-7.

10. Allender S, Owen B, Kuhlberg J, et al. A Community Based Systems Diagram of Obesity Causes. PLoS One 2015;10:e0129683.

11. Friel S, Pescud M, Malbon E, et al. Using systems science to understand the determinants of inequities in healthy eating. PLOS One 2017;12:e0188872.

12. Green L, Sim L, Breiner H. Evaluating Obesity Prevention Efforts: A Plan for Measuring Progress: National Academies Press, 2013. Available from. https://www.ncbi.nlm.nih.gov/books/NBK202498/.

13. Rosas SR. Systems thinking and complexity: considerations for health promoting schools. Health Promot Int 2017;32:dav109. 
14. Carey G, Malbon E, Carey N, et al. Systems science and systems thinking for public health: a systematic review of the field. BMJ Open 2015;5:e009002.

15. Peters $\mathrm{DH}$. The application of systems thinking in health: why use systems thinking? Health Res Policy Syst 2014;12:51.

16. Mabry PL, Marcus SE, Clark PI, et al. Systems science: a revolution in public health policy research. Am J Public Health 2010;100:1161-3.

17. Luke DA, Stamatakis KA. Systems science methods in public health: dynamics, networks, and agents. Annu Rev Public Health 2012;33:357-76.

18. Adam T. Advancing the application of systems thinking in health Health Res Policy Syst 2014;12:50.

19. Swanson RC, Cattaneo A, Bradley E, et al. Rethinking health systems strengthening: key systems thinking tools and strategies for transformational change. Health Policy Plan 2012;27 Suppl 4:iv54-iv61.

20. Kothari A, Hamel N, MacDonald J-A, et al. Exploring Community Collaborations: Social Network Analysis as a Reflective Tool for Public Health. Syst Pract Action Res 2014;27:123-37.

21. Hawe P, Webster C, Shiell A. A glossary of terms for navigating the field of social network analysis. $J$ Epidemiol Community Health 2004;58:971-5.

22. Chronic Disease Prevention Directorate. Western Australian Health Promotion Strategic Framework 2017-2021. Perth: Western Australia, 2017. Department of Health Western Australia

23. The Australian Prevention Partnership Centre. The structure of Prevention Tracker. Sax Institute 2018.

24. Cleland V, McNeilly B, Crawford D, et al. Obesity prevention programs and policies: practitioner and policy-maker perceptions of feasibility and effectiveness. Obesity 2013;21:n/a-E55.

25. Aicken $\mathrm{C}$, Roberts $\mathrm{H}$, Arai L. Mapping service activity: the example of childhood obesity schemes in England. BMC Public Health 2010;10:310.

26. Nichols MS, Reynolds RC, Waters E, et al. Community-based efforts to prevent obesity: Australia-wide survey of projects. Health Promot J Austr 2013;24:111-7.

27. The Australian Prevention Partnership Centre. A community snapshot. Sax Institute 2018.

28. The Australian Prevention Partnership Centre. System Inventory. Sax Institute 2018.
29. The Australian Prevention Partnership Centre. Social network analysis. Sax Institute 2018.

30. Davidson S, Morgan M. Systems Change Framework. Hobart: Sax Institute, 2018.

31. Richter M, Dragano N. Micro, macro, but what about meso? The institutional context of health inequalities. Int J Public Health 2018;63:163-4.

32. Lee-Han H, Dwyer J, Johnson I. Application of Nominal Group Technique to evaluate a community health status report. The Canadian Journal of Program Evaluation 1996;11.

33. Biernacki P, Waldorf D. Snowball Sampling: Problems and Techniques of Chain Referral Sampling. Sociol Methods Res 1981:10:141-63.

34. Australian Health Practitioner Regulation AgencyRegistration Codes and guidelines 2018 https://www.ahpra.gov.au/Registration/ Registration-Standards/codes-guidelines.aspx.

35. Corp. I. IBM SPSS Statistics for Windows, Version 25.0. Arnmork NY: IMB Corp, 2017.

36. PARTNER Tool. Program to Analyze, Record, and Track Networks to Enhance Relationships. 2012 http://www.partnertool.net (cited 2018 08 Feb,).

37. Milward HB, Provan KG, Structure MNPublic Adm 1998;76:387-407.

38. Borgatti SP, Carley KM, Krackhardt D. On the robustness of centrality measures under conditions of imperfect data. Soc Networks 2006;28:124-36.

39. Qualtrics. Qualtrics. Provo, Utah, USA, 2018.

40. Borgatti S, Everett M, Freeman L. Ucinet for Windows: Software for social network analysis. Analytic Technologies: Harvard, 2002.

41. Borgatti S. NetDraw: Graph visualization software. Harv Analytic Technologies 2002.

42. Rombach MP, Porter MA, Fowler JH, et al. Core-Periphery Structure in Networks. SIAM J Appl Math 2014;74:167-90.

43. Home Office. Social Network Analysis: 'How to guide', 2016.

44. Freeman LC. Centrality in social networks conceptual clarification. Soc Networks 1978;1:215-39.

45. Brownson RC, Colditz GA, Proctor EK, Eds. Dissemination and implementation research in health: translating science to practice / edited by. Oxford; New York: Oxford University Press, 2012. 